\title{
Crystal Structure of the DH/PH Fragment of Dbs without Bound GTPase
}

\author{
David K. Worthylake, Kent L. Rossman, \\ and John Sondek* \\ Department of Pharmacology and \\ Department of Biochemistry and Biophysics and \\ The Lineberger Comprehensive Cancer Center \\ University of North Carolina School of Medicine \\ Chapel Hill, North Carolina 27599
}

\section{Summary}

Dbl proteins are guanine nucleotide exchange factors for Rho GTPases, containing adjacent Dbl homology (DH) and pleckstrin homology (PH) domains. This domain architecture is virtually invariant and typically required for full exchange potential. Several structures of DH/PH fragments bound to GTPases implicate the $\mathrm{PH}$ domain in nucleotide exchange. To more fully understand the functional linkage between $\mathrm{DH}$ and $\mathrm{PH}$ domains, we have determined the crystal structure of the DH/PH fragment of Dbs without bound GTPase. This structure is generally similar to previously determined structures of Dbs bound to GTPases albeit with greater apparent mobility between the DH and PH domains. These comparisons suggest that the $\mathrm{DH}$ and $\mathrm{PH}$ domains of Dbs are spatially primed for binding GTPases and small alterations in intradomain conformations that may be elicited by subtle biological responses, such as altered phosphoinositide levels, are sufficient to enhance exchange by facilitating interactions between the PH domain and GTPases.

\section{Introduction}

The 20 members of the Rho family of small GTPases regulate a variety of diverse cellular processes many of which affect the structure of the actin-based cytoskeleton (Etienne-Manneville and Hall, 2002; Ridley, 2001). Like other small GTPases and heterotrimeric G proteins, Rho proteins function as molecular switches: active when bound to GTP and inactive when bound to GDP. Activation is catalyzed by guanine nucleotide exchange factors (GEFs) that directly bind Rho GTPases to promote the dissociation of GDP and the loading of GTP. Conversely, GTPase activating proteins (GAPs) antagonize downstream signaling by preferentially binding GTP-bound Rho GTPases to promote enhanced GTP hydrolysis.

Dbl family members constitute the largest group of GEFs specific for Rho GTPases (Whitehead et al., 1997). The first identified member of this family, Dbl, was discovered as a transforming gene during transfection experiments using DNA derived from a diffuse $b$ cell lymphoma (Eva et al., 1988). Since the discovery of Dbl, this family has grown to include over 60 distinct human proteins characterized by a $\sim 200$ residue Dbl homology

*Correspondence: sondek@med.unc.edu
(DH) domain followed almost invariably by a $\sim 100$ residue pleckstrin homology $(\mathrm{PH})$ domain. Outside of the characteristic $\mathrm{DH}$ and $\mathrm{PH}$ domains, family members are highly diverse in sequence.

Nucleotide exchange activity primarily resides within the $\mathrm{DH}$ domain and recently determined structures of the $\mathrm{DH} / \mathrm{PH}$ fragments from T-lymphoma invasion and metastasis factor (Tiam1), Dbl's big sister (Dbs), and intersectin (ITSN) in complex with their cognate Rho GTPases (Rossman et al., 2002; Snyder et al., 2002; Worthylake et al., 2000) indicate a highly conserved exchange mechanism. In brief, the conformationally variable switch regions of the Rho GTPases engage two highly conserved regions (CR1 and CR3) within the DH domains to fix the switch regions in conformations energetically unfavorable for nucleotide binding. Relative to GDP concentrations, high levels of GTP drive the loading of GTP onto bound GTPases, leading to dissociation of GEF-GTPase complexes and subsequent downstream signaling by GTP-bound GTPases.

While these structural studies and numerous complementary functional studies emphasize the importance of DH domains in engaging GTPases for functional exchange, roles for the associated $\mathrm{PH}$ domains remain poorly understood. Many PH domains bind phosphoinositides with low affinity and specificity (Lemmon and Ferguson, 2000), and most suggestions for functional roles of $\mathrm{DH}$-associated $\mathrm{PH}$ domains have involved lipid binding either to regulate the intracellular location of $\mathrm{Dbl}$ family proteins (Whitehead et al., 1999) or to allosterically modulate the $\mathrm{DH} / \mathrm{PH}$ cassette irrespective of other intracellular components (Crompton et al., 2000; Han et al., 1998; Russo et al., 2001). However, one intriguing possibility is that $\mathrm{DH}$-associated $\mathrm{PH}$ domains actively participate in the exchange process by directly interacting with cognate GTPases. This situation is clearly operative for Dbs where the crystal structures highlight direct interactions between the GTPases and the PH domain that when destroyed by mutation severely diminish nucleotide exchange in vitro and in vivo while preserving the overall fold and lipid binding potential of mutated Dbs (Rossman et al., 2002, 2003). However, in contrast to Dbs, the crystal structures of the $\mathrm{DH} / \mathrm{PH}$ fragments of Tiam1 and ISTN show no direct interactions between the PH domains and bound GTPases. Furthermore, in the crystal structure of the DH/PH portion of Sos1 without a bound GTPase, the PH domain actually blocks the conserved face of the $\mathrm{DH}$ domain necessary for engaging GTPases (Soisson et al., 1998).

To account for the seemingly disparate and contradictory biochemical and structural data regarding functional roles for $\mathrm{DH}$-associated $\mathrm{PH}$ domains, we have proposed a general model linking the binding of membrane-resident phosphoinositides through the $\mathrm{PH}$ domain with the regulation of the conformational heterogeneity between $\mathrm{DH}$ and $\mathrm{PH}$ domains to modulate the exchange reaction (Rossman et al., 2002). Put simply, binding of phosphoinositides by the $\mathrm{PH}$ domain would restrict the conformational heterogeneity between $\mathrm{DH}$ 
and $\mathrm{PH}$ domains forced to operate in close proximity to two-dimensional lipid bilayers. These spatial restrictions would then favor direct interactions between the $\mathrm{PH}$ domains and cognate GTPases leading to elevated nucleotide exchange. There is increasing evidence to support such a model. For example, mutations within the $\mathrm{DH}$-associated PH domains of Tiam1 and Dbs that abrogate phosphoinositide binding while preserving nucleotide exchange in vitro as well as proper intracellular targeting, nonetheless fail to activate their cognate GTPases in vivo upon overexpression in cell culture (Baumeister et al., 2003; Rossman et al., 2003). Similarly, in transgenic worms expressing the Dbl family member UNC-73 mutated within the PH domain to destroy lipid binding but not intrinsic nucleotide exchange in vitro, mutated UNC-73 is properly localized but cannot rescue neuronal abnormalities associated with a gross deletion of wild-type UNC-73 (Kubiseski et al., 2003).

In order to better understand the interplay between $\mathrm{DH}$ and $\mathrm{PH}$ domains, we have determined the crystal structure of the $\mathrm{DH} / \mathrm{PH}$ fragment of Dbs without a bound GTPase. In general, the DH/PH portion of unbound Dbs is similar, though not identical, to the equivalent fragment of Dbs bound to either RhoA or Cdc42. Importantly, unbound Dbs can easily accommodate the binding of a GTPase without massive interdomain rearrangements and does not adopt a conformation similar to unbound Sos1 possessing a PH domain that occludes the GTPase binding surface of the DH domain. The crystal structure of unbound Dbs provides four independent representations of the DH/PH fragment, and comparison of these molecules reveal a high degree of conformational variability between $\mathrm{DH}$ and $\mathrm{PH}$ domains absent from GTPasebound forms of Dbs. Presumably for Dbs and other Dbl family members that readily activate GTPases, the DH and $\mathrm{PH}$ domains are spatially and conformationally primed for productive interaction with GTPases. This suggestion is further supported by the recent structure determination of the N-terminal DH/PH region from Trio without bound GTPase (coordinates generously provided by $\mathrm{N}$. Nassar et al., personal communication) highlighting a remarkably similar overall conformation to Dbs. Subsequent restrictions in the intrinsic conformational heterogeneity between $\mathrm{DH}$ and $\mathrm{PH}$ domains as exemplified for Dbs could thus be used to regulate the exchange process through direct participation of the $\mathrm{PH}$ domain consistent with a conserved functional linkage between $\mathrm{DH}$ and $\mathrm{PH}$ domains operating at lipid membranes.

\section{Results and Discussion}

\section{Overall Structure}

There are four independent representations of the DH/ $\mathrm{PH}$ fragment of Dbs within the crystal asymmetric unit (molecules A-D), two of which are shown in Figure 1. The crystal form used to determine the structure has high intrinsic disorder as exemplified by a high Wilson temperature factor (see Experimental Procedures) and extreme care was taken in building the final model. For example, the top panel in Figure 1 represents a composite simulated-annealing $2 \mathrm{Fo}-\mathrm{Fc}$ omit map surrounding molecule $D$. This molecule has the highest average temperature factor $\left(124 \AA^{2}\right)$ of the four representations within the asymmetric unit and represents the portion of the final model with the largest degree of molecular disorder and associated experimental uncertainty. Nonetheless, the model for molecule $D$ is a reasonable interpretation of the electron density and in general resembles the more ordered portions of the final model (Figure 1 bottom panel; molecule $A$ with mean temperature factor of $65 \AA^{2}$ ).

The final model for the DH/PH fragment of Dbs is remarkably similar in overall conformation to previously determined structures of this fragment bound to either Cdc42 or RhoA (Rossman et al., 2002; Snyder et al., 2002) and contrasts dramatically with the equivalent portion of Sos1 without a bound GTPase (Figure 2). GTPases primarily engage conserved regions 1 and 3 within DH domains (Figures 2 and 3 ), and these regions are freely available to incoming GTPases in the unbound version of Dbs. In contrast, the $\mathrm{PH}$ domain of Sos1 occludes conserved regions 1 and 3 . This arrangement of $\mathrm{DH}$ and $\mathrm{PH}$ domains is expected to hinder efficient guanine nucleotide exchange and indeed this portion of Sos1 is incompetent for exchange when assayed in vitro (S. Soisson, personal communication). Presumably, unknown intracellular conditions regulate the removal of the $\mathrm{PH}$ domain from the conserved surface of the $\mathrm{DH}$ domain within Sos1 to promote exchange activity on Rac, the pertinent GTPase activated in vivo by Sos1 (Nimnual et al., 1998; Scita et al., 1999, 2001).

The four molecules have associated into two similar, though not identical, head-to-tail dimers. The GTPase binding surfaces of all DH domains are freely accessible. However, within each dimer, the DH domains are roughly perpendicular suggesting that the prenylated $\mathrm{C}$ termini of two bound GTPases would be unable to insert simultaneously into a membrane. Furthermore, while one of the $\mathrm{PH}$ domains within each dimer would be able to engage a membrane utilizing positively charged residues in the $\beta 1 / \beta 2$ and $\beta 3 / \beta 4$ loops, these residues in the other $\mathrm{PH}$ domain are buried within the dimer interface. Therefore, although each dimer buries $\approx 1250 \AA^{2}$ of solvent-accessible surface area, the above considerations make it unlikely that the Dbs dimers seen here are physiologically relevant.

\section{GTPase-free Dbs Displays Interdomain Heterogeneity} There are four independent representations per asymmetric unit for both unbound Dbs as well as Dbs bound to RhoA, providing a unique opportunity to assess internal motions. Upon superposition of the Dbs fragments in each crystal form, it is readily apparent that RhoA dramatically restricts the $\mathrm{DH}$ and $\mathrm{PH}$ domains to essentially one conformation (Figure 4; see Table 1). Conversely, lack of a bound GTPase allows for a relatively large degree of conformational heterogeneity between $\mathrm{DH}$ and PH domains. This heterogeneity initiates within the $\alpha 6$ helix of the $\mathrm{DH}$ domain and propagates C-terminal into the $\mathrm{PH}$ domain where the initial relatively minor differences in structure are considerably amplified. As described previously (Rossman et al., 2002; Snyder et al., 2002), RhoA and Cdc42 interact with both the $\alpha 6$ 

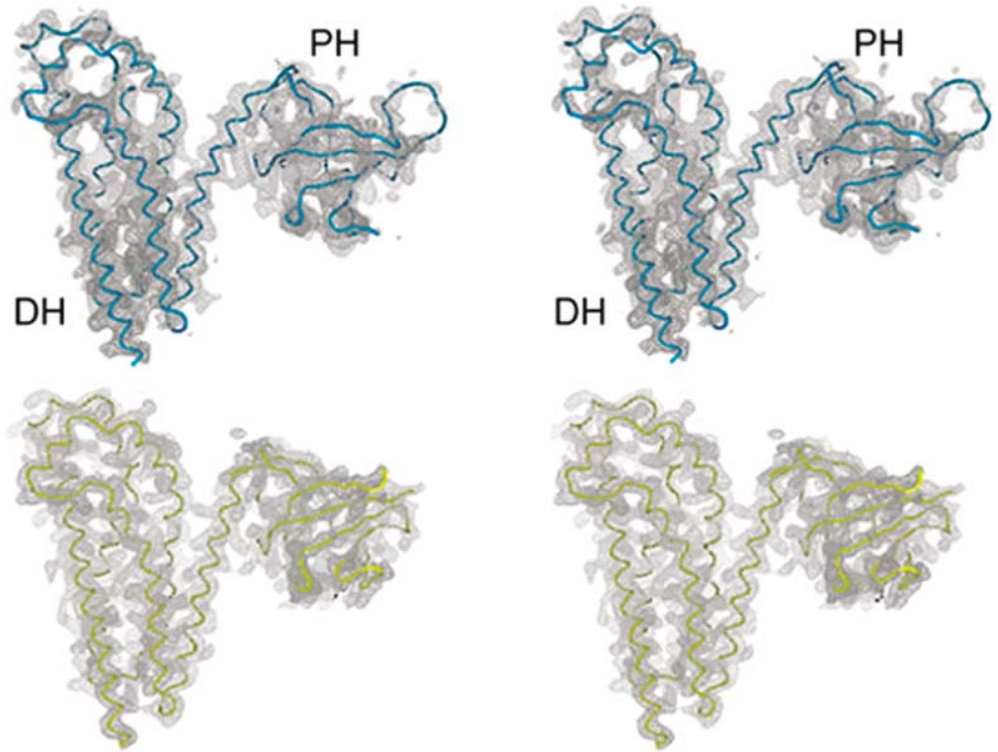

Figure 1. Stereoview Omit and 2Fo-Fc Electron Densities

Top, a composite simulated-annealing 2FoFc omit map was calculated by iteratively omitting $5 \%$ of the final model, then submitting the coordinates to $1000 \mathrm{~K}$ torsion-angle simulated-annealing refinement prior to map generation. The map shown is contoured at $1 \sigma$ and surrounds a worm representation of molecule $D$ (mean temperature factor $124 \AA^{2}$ ). Bottom, 2Fo-Fc density map calculated using phases derived from the final model, surrounds molecule $A$ (mean temperature factor $65 \AA^{2}$ ). Map is contoured at $1.5 \sigma$. Figure was made using SPOCK (Christopher, 1998). helix of the $\mathrm{DH}$ domain and the $\beta 3 / \beta 4$ loop of the $\mathrm{PH}$ domain. Clearly, these interactions serve not only to reorient the $\mathrm{PH}$ domain relative to the $\mathrm{DH}$ domain, but also serve to restrict the conformational heterogeneity inherent between these domains. The conformational heterogeneity of the four $\mathrm{DH}$ and $\mathrm{PH}$ domains within the asymmetric unit of Tiam1/Rac1 (data not shown) are also restricted relative to the unliganded fragment of Dbs, presumably due to interactions between Rac1 and $\alpha 6$ of Tiam1.

GTPase Binding Reorients the PH Domain of Dbs While the structures of Dbs either bound to GTPase or unbound are similar, they are not identical, and the differences between the two forms are important for understanding the molecular aspects of guanine nucleotide exchange catalyzed by the concerted action of the $\mathrm{DH}$ and $\mathrm{PH}$ domains. In particular, binding of either RhoA or Cdc42 leads to the ordering of the $\beta 3 / \beta 4$ loop of the $\mathrm{PH}$ domain and a concerted rotation of the $\mathrm{PH}$ domain relative to the $\mathrm{DH}$ domain about the $\alpha 6$ helix of the $\mathrm{DH}$ domain (Figures 3 and 4). RhoA and Cdc42 directly interact with both the $\beta 3 / \beta 4$ loop and the $\alpha 6$ helix of Dbs (Rossman et al., 2002; Snyder et al., 2002), and these interactions are presumably the major immediate determinants dictating the placement of these structural features as well as the final relative disposition of the $\mathrm{DH}$ and $\mathrm{PH}$ domains. The importance of $\mathrm{PH}$ domain

\section{GTPase-free Dbs}

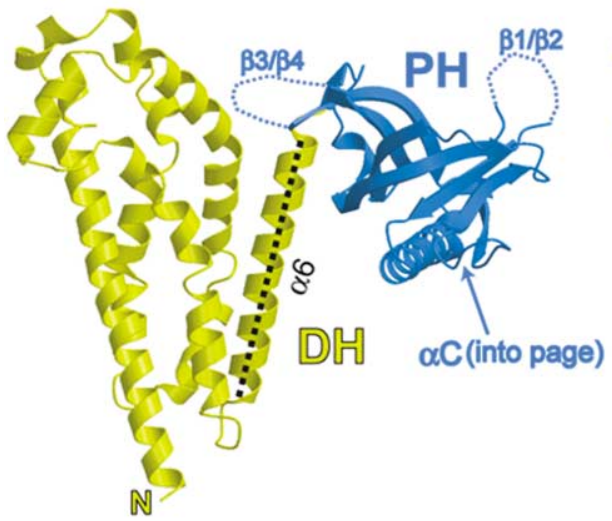

Dbs from complex

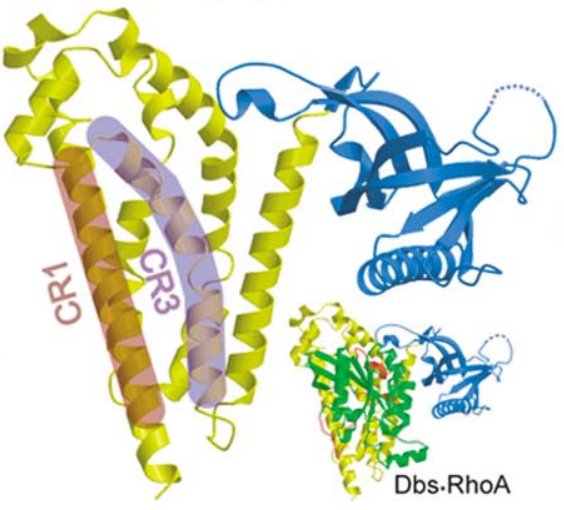

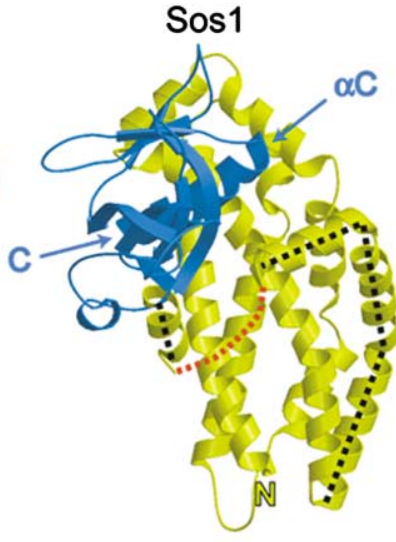

Figure 2. Schematic Representations of the DH/PH Domains from Three Structures

Left, the DH (residues 624-818) and PH domains (residues 819-958) of molecule A of isolated Dbs have been colored yellow and blue respectively. Missing residues in PH domain loops are represented as blue dashed lines. Helix $\alpha 6$ is highlighted with black dashed line. Center, Dbs from the Dbs $\cdot$ RhoA structure (PDB accession 1LB1) has been aligned with isolated Dbs using DH domain residues. Conserved regions 1 and 3 are highlighted with transparent cylinders. In both the Dbs $\cdot \mathrm{RhoA}$ and Dbs $\cdot \mathrm{Cdc} 42$ structures, the $\beta 3 / \beta 4$ loop of the $\mathrm{PH}$ domain is stabilized by contact with the GTPase (inset, green with switch regions in red). Right, the DH/PH fragment of Sos1 (PDB accession 1DBH) was aligned relative to unbound Dbs using the conserved regions ( $r m s d=1.8 \AA$ for $72 \mathrm{C} \alpha$ atoms). The structural elements equivalent to $\alpha 6$ in $\mathrm{Dbs}$ is highlighted with a black dashed line. Disordered residues in Sos1 are represented by a red dashed line. Note that the dramatically different orientation of the Sos PH domain partially occludes the DH domain and prohibits GTPase binding. 


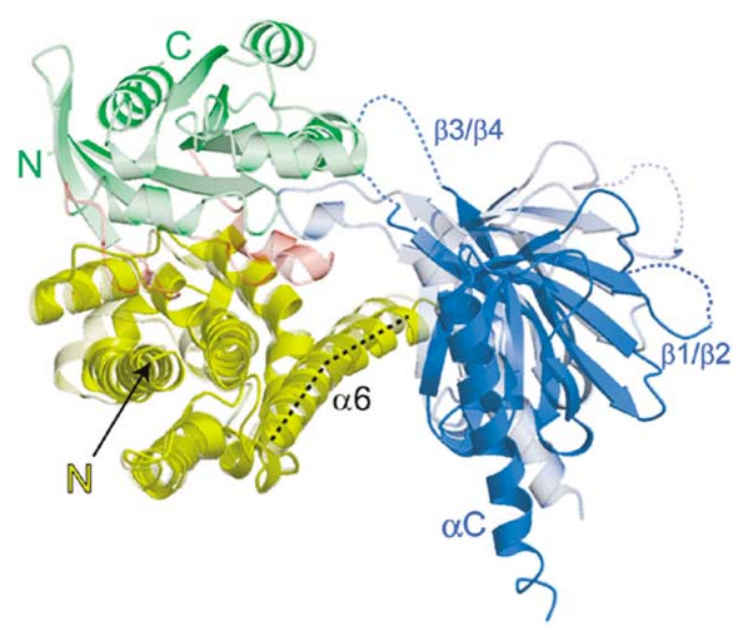

Figure 3. Conformational Changes Induced within the DH/PH Domains of Dbs upon Binding a GTPase

The structure of Dbs•RhoA (transparent) was superimposed on the GTPase-free form of Dbs (molecule B) using $\mathrm{DH}$ domain residues. Significant interactions between RhoA and the $\beta 3 / \beta 4$ loop as well as $\alpha 6$ serve to reduce the conformational heterogeneity between $\mathrm{DH}$ and $\mathrm{PH}$ domains and result in the ordering of the $\beta 3 / \beta 4$ loop and the tilting of the PH domain toward the GTPase.

contacts is supported by the observation that Tyr889, within the $\beta 3 / \beta 4$ loop of the PH domain, when mutated to phenylalanine destroys specific interactions with Cdc42 and RhoA and results in a slight reorientation of the $\mathrm{PH}$ domain relative to the $\mathrm{DH}$ domain (Rossman et al., 2002). In the structure of Dbs(Y889F) bound to Cdc42, the relative position of the $\mathrm{DH}$ and $\mathrm{PH}$ domains is approximately intermediate between the unbound form of $\mathrm{Dbs}$ and wild-type Dbs bound to either Cdc42 or RhoA.

\section{Heterogeneity within the DH and PH Domains}

Relative to Dbs bound to GTPases, there is a small amount of disorder within the superimposed $\mathrm{DH}$ domains of unbound Dbs (Figure 4). This disorder is localized to the amino terminus and portions of the $\mathrm{DH}$ domain previously described as the "seatback." The seatback protrudes from the body of the DH domain and provides the majority of structural determinants dictating specificity for the productive engagement of the various Rho GTPases. Intradomain interactions link the seatback to $\alpha 6$ of the $\mathrm{DH}$ domain, and the binding of GTPases would also stabilize $\alpha 6$ indirectly through stabilization of the seatback.

Upon superposition, it is also evident that the four $\mathrm{PH}$ domains within the crystal structure of the fragment of unbound Dbs are also highly similar, excluding of course the highly mobile loops connecting some secondary structure elements (Figure 4).

\section{Conclusions}

There are now several structures of individual DH domains (Aghazadeh et al., 1998, 2000; Liu et al., 1998), as well as structures of the DH/PH fragment of Sos 1 (Soisson et al., 1998) and several equivalent regions of other Dbl family GEFs bound to their cognate GTPases (Rossman et al., 2002; Snyder et al., 2002; Worthylake et al., 2000). However, until now we have lacked a matched pair of structures, that is, an isolated fragment and a GTPase bound version from the same Dbl family protein that would allow direct comparison of alterations directly related to the engagement of a GTPase by a Dbl family protein. We have rectified this problem here with the presentation of the $3 \AA$ crystal structure of the isolated $\mathrm{DH} / \mathrm{PH}$ fragment of $\mathrm{Dbs}$ that we have compared with the preexisting structures of this identical $\mathrm{DH} / \mathrm{PH}$ fragment bound to either RhoA or Cdc42. In general, the isolated DH/PH portion of Dbs is remarkably similar in overall conformation to the equivalent region of $\mathrm{Dbs}$ bound to either Cdc42 or RhoA (Rossman et al., 2002; Snyder et al., 2002). This observation is somewhat surprising given the dramatically different arrangement of the $\mathrm{DH}$ and $\mathrm{PH}$ domains in the equivalent fragment of Sos1 also not bound to a GTPase (Soisson et al., 1998). Within the structure of Sos1, the PH domain occludes the surface within the DH domain necessary for productive engagement of GTPases and, consistent with biochemical data, this fragment of Sos1 cannot productively engage Rac1 in vitro (S. Soisson, personal communication) nor in vivo without coexpression of Ras (Nimnual et al., 1998). Since Rac1 is activated in vivo by full-length Sos1, presumably other cellular factors such as Eps8 and E3b1 (Innocenti et al., 2002; Nimnual and Bar-Sagi, 2002; Scita et al., 1999) are required to alter the relative orientation of the $\mathrm{DH}$ and PH domains to allow free access to the GTPase binding surface within Sos1. In comparison, the structure of the unbound form of Dbs highlights a DH domain easily accessible to GTPases, and no large rearrangements of the $\mathrm{PH}$ domain with respect to the $\mathrm{DH}$ domain are required for productive engagement of GTPases. On the contrary, the isolated $\mathrm{DH} / \mathrm{PH}$ fragment of $\mathrm{Dbs}$ is remarkably similar in overall interdomain arrangement to its GTPases-bound equivalents, suggesting that the $\mathrm{DH}$ and PH domains of Dbs are spatially primed to engage GTPases. Moreover, in results shared before publication, N. Nassar et al. have determined the crystal structure of a DH/PH fragment from Trio. Because there is only one copy of the Trio $\mathrm{DH} / \mathrm{PH}$ fragment in the crystal asymmetric unit, conformational heterogeneity is not observed in that structure. Nevertheless, the single Trio DH/PH fragment features an arrangement of domains remarkably similar to the unbound form of Dbs. These results suggest that inhibition of nucleotide exchange by occlusion of the $\mathrm{DH}$ domain by the $\mathrm{PH}$ domain as exemplified by Sos1 may not be a general regulatory mechanism.

While the GTPase-bound and unbound versions of the $\mathrm{DH} / \mathrm{PH}$ fragment of Dbs are similar, they are not identical, and the fortunate occurrence of multiple independent representations per asymmetric unit in the various crystals forms of Dbs provides a high degree of confidence in conclusions derived from these differences. In particular, the unbound version of Dbs demonstrates a high degree of intrinsic mobility between and within domains not observed in the GTPase-bound versions. This increased mobility manifests as conformational heterogeneity in regions necessary for the productive engagement of GTPases. More specifically, for the four independent representations of unbound $\mathrm{Dbs}$, the $\mathrm{DH}$ and $\mathrm{PH}$ domains occupy a variety of subtly different 


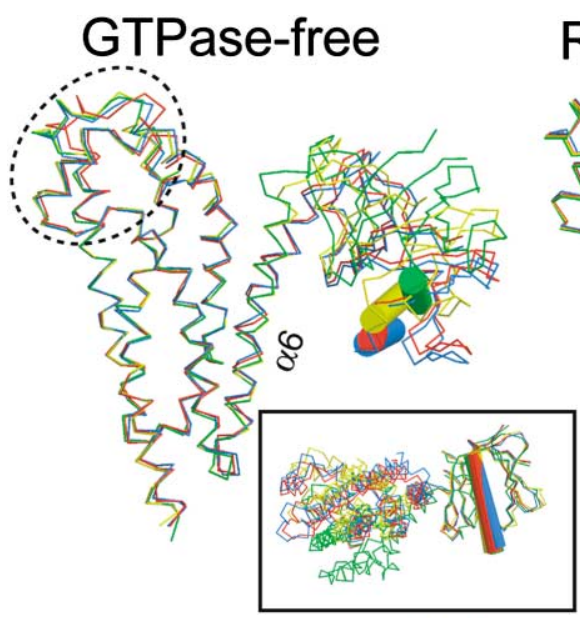

RhoA bound
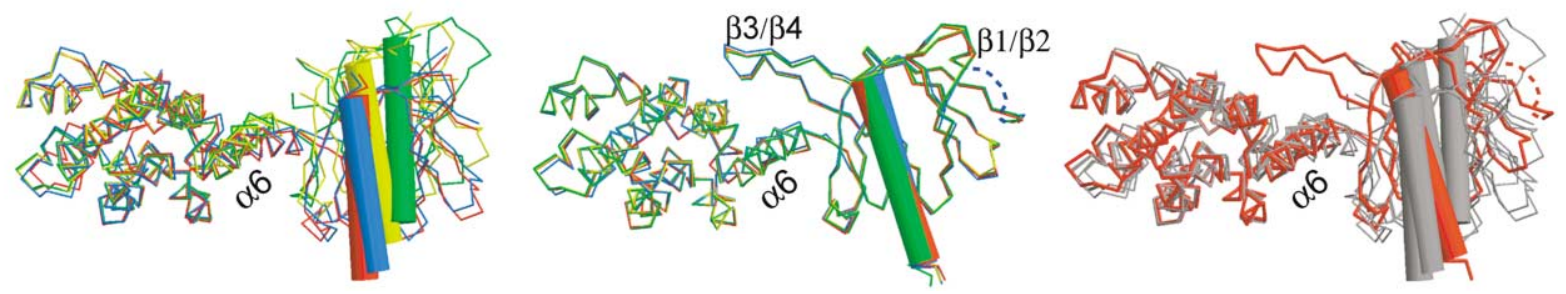

Figure 4. Superimposed DH/PH Fragments from Isolated Dbs, the Dbs•RhoA Complex, and Isolated Dbs with $\mathrm{Dbs} \cdot \mathrm{RhoA}$

Left, the four GTPases-free Dbs fragments within the crystal asymmetric unit were superimposed using $\mathrm{DH}$ domain residues and shown in two orientations. The seatback described in the text has been circled with a dashed line. For simplicity, only common residues are shown (residues 624-842, 857-876, and 889-950). Residues 932-950 of $\alpha \mathrm{C}$ of the PH domains are represented as cylinders. Inset, the four molecules were superimposed using common $\mathrm{PH}$ domain residues (C $\alpha$ atoms 828-842, 857-876, and 889-950) and oriented similar to the bottom orientation. Note that while the intradomain conformations are highly similar, there is a large degree of interdomain variability with molecule $\mathrm{C}$ (green) representing an extreme. Center, the Dbs ${ }^{\circ} \mathrm{RhoA}$ structure was submitted to $5000^{\circ}$ simulated-annealing refinement using torsion angle dynamics without restraints on noncrystallographic symmetry. The four DH/PH fragments from this crystal asymmetric unit were then superimposed and oriented similar to the previous example. Note that in contrast to the isolated fragments of Dbs, binding of RhoA induces essentially one conformation. Right, the four GTPase-free Dbs molecules (gray) were aligned with the DH/PH fragment of the Dbs $\cdot \mathrm{RhoA}$ (complex A; red). Note that none of the GTPase-free Dbs molecules exactly duplicates the interdomain conformation seen in the Dbs $\cdot R$ hoA complex.

relative orientations. These differences arise within the C-terminal region of the $\mathrm{DH}$ domain $(\alpha 6)$ that forms part of the conserved interface with GTPases, and the differences become exaggerated as they propagate into the $\mathrm{PH}$ domain. Within the $\mathrm{PH}$ domain, the $\beta 3 / \beta 4$ loop that directly contacts GTPases for productive exchange is disordered. Similarly, the seat-back within the DH domain that directly contacts GTPases and partially dictates the specificity for pairing of GTPases and Dbl family proteins occupies several alternate conformations. It seems clear that the binding of either RhoA or Cdc42 to Dbs restricts the conformational heterogeneity of the
$\mathrm{DH}$ and $\mathrm{PH}$ domains, and we have used this information to support and extend our previous interpretations designed to integrate the functions of paired $\mathrm{DH}$ and $\mathrm{PH}$ domains operating at membrane surfaces for the efficient activation of Rho GTPases (Figure 5).

In this model, the paired $\mathrm{DH}$ and $\mathrm{PH}$ domains of Dbs possess high intrinsic mobility that limit but do not hinder the efficient activation of Rho GTPases. The DH domain is free to engage a membrane-bound GTPase through its conserved binding interface, but critical interactions with the $\mathrm{PH}$ domain are not sufficiently stable to provide physiologically meaningful guanine nucleo-

Table 1. Root-Mean-Square Differences of Superimposed Structures

\begin{tabular}{|c|c|c|c|c|c|c|c|c|c|}
\hline & \multicolumn{3}{|c|}{ DH Domains $^{a}(\AA ̊)$} & \multicolumn{3}{|c|}{ PH Domains $(\AA ̊)$} & \multicolumn{3}{|c|}{ All Common $(\AA)$} \\
\hline & Mean $^{b}$ & High & Low & Mean & High & Low & Mean & High & Low \\
\hline GTPase-free & 1.09 & 1.39 & 0.51 & 1.36 & 1.94 & 0.73 & 2.95 & 4.71 & 1.09 \\
\hline RhoA bound & 0.27 & 0.39 & 0.15 & 0.32 & 0.42 & 0.23 & 0.34 & 0.48 & 0.21 \\
\hline GTPase-free versus RhoA bound & 0.99 & 1.35 & 0.60 & 1.06 & 1.90 & 0.65 & 2.16 & 3.75 & 1.18 \\
\hline
\end{tabular}

${ }^{\text {a }}$ Rmsd is calculated for sets of superimposed $\mathrm{C} \alpha$ atoms: (DH domain, 624-817; PH domain, 818-842, 857-876, and 889-950; all common, 624-842, 857-876, and 889-950).

${ }^{b}$ For individual crystal forms, mean values are calculated using the six unique superpositions for the four molecules per asymmetric unit. Between crystal forms, mean values are calculated from the four superpositions of each GTPase-free molecule onto the DH/PH portion of complex A of the RhoA-bound form. 


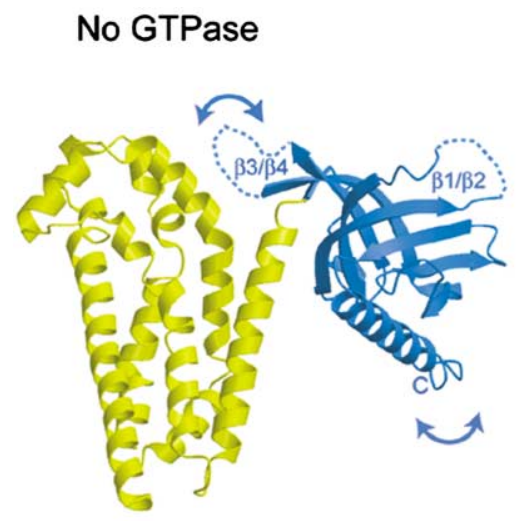

\section{Sub-optimal binding and exchange}

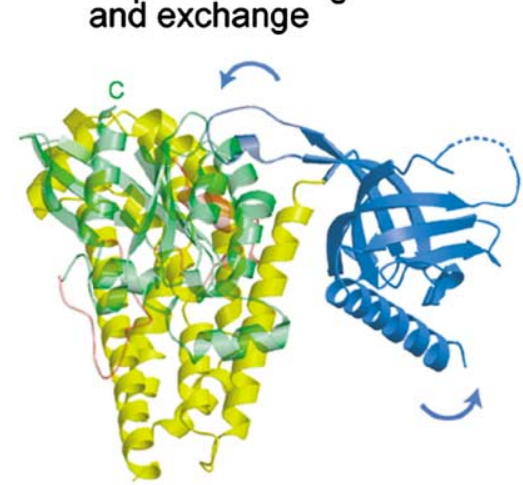

\section{Full binding and exchange}

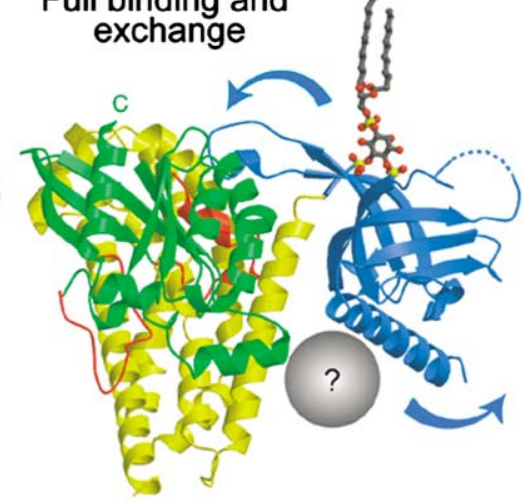

Figure 5. Activity States of Dbl GEFs

The $\mathrm{DH}$ and $\mathrm{PH}$ domains of Dbs exhibit a large degree of intrinsic conformational heterogeneity between the domains (left, double arrowheads) and within the PH domain as indicated by the lack of ordered $\beta 1 / \beta 2$ and $\beta 3 / \beta 4$ loops (dotted lines). Binding of membrane resident RhoA or Cdc42 (center) severely restricts conformational heterogeneity and slightly reorients the $\mathrm{PH}$ domain toward the $\mathrm{DH}$ domain (single arrowheads). However, full exchange potential of Dbs in vivo is not realized without the binding of inositol phospholipids within membranes (Rossman et al., 2003), suggesting that further conformational restrictions are imposed by membrane surfaces (right panel). General location and orientation of $\mathrm{PI}(4,5) \mathrm{P}_{2}$ docked to the $\mathrm{PH}$ domain and based upon previous analyses (Ferguson et al., 2000; Rossman et al., 2002) is shown schematically. Substantial biochemical data for other Dbl-family proteins (i.e., Tiam1 [Baumeister et al., 2003] and UNC-73 [Kubiseski et al., 2003]) support similar regulatory mechanisms in these family members, suggesting that such regulation may be generally operable in Dbl family GEFs. Alternatively, positive or negative regulation can occur through binding of a cellular factor (gray sphere) that affects the relative orientations of the DH and PH domains. Figures 2-5 were made using Molscript and Raster3d (Kraulis, 1991; Merritt and Murphy, 1994).

tide exchange. However, high intrinsic conformational heterogeneity between $\mathrm{DH}$ and $\mathrm{PH}$ domains as well as within the loops of the $\mathrm{PH}$ domain can be restricted through the binding of membrane-resident phosphatidylinositide 4,5-bisphosphate $\left(\mathrm{PI}(4,5) \mathrm{P}_{2}\right)$ to the $\mathrm{PH}$ domain. This restricted conformational heterogeneity is sufficient to stabilize a limited number of specific interactions between the PH domain and GTPases necessary for efficient exchange. In particular, interactions between the $\beta 3 / \beta 4$ loop of the $\mathrm{PH}$ domain and Rho GTPases previously shown to be critical for efficient exchange would be stabilized (Rossman et al., 2002). Similarly, it also seems likely that the conformational heterogeneity of the $\alpha 6$ helix within the $C$ terminus of the $\mathrm{DH}$ domain would also be dependent on the relative freedom of the linked $\mathrm{PH}$ domain and restricting the conformational space accessible to the $\mathrm{DH} / \mathrm{PH}$ cassette through lipid binding to the $\mathrm{PH}$ domain may favor re- stricted forms of $\alpha 6$ that enhance exchange. In this manner, the DH and PH domains of Dbs function together to provide highly regulated activation of GTPases dependent upon the presence of both membrane-resident GTPases and $\mathrm{PI}(4,5) \mathrm{P}_{2}$. It seems likely that such a model is also operative for other Dbl family proteins such as Tiam1 (Baumeister et al., 2003) and UNC-73 (Kubiseski et al., 2003) where lipid binding to the $\mathrm{PH}$ domain has been shown to be necessary for biological activities associated with the efficient activation of cognate GTPases but not required for proper intracellular targeting of the exchange factors.

In a variant of this model, it seems reasonable to suggest that other intracellular conditions capable of altering the conformational heterogeneity between paired $\mathrm{DH}$ and $\mathrm{PH}$ domains could also regulate guanine nucleotide exchange. For instance, $\mathrm{G} \alpha 13$ directly interacts with both the DH/PH portion and the RGS domain

\begin{tabular}{|c|c|c|c|c|c|}
\hline \multicolumn{6}{|l|}{ Data Statistics } \\
\hline Wavelength $(\AA)$ & Resolution (shell) (Å) & $\begin{array}{l}\text { Observations } \\
\text { (Total/Unique) }\end{array}$ & Completeness (\%) & $<\mathrm{l} / \sigma \mathrm{l}>^{\mathrm{a}}$ & $\mathbf{R}_{\text {sym }}{ }^{b}(\%)$ \\
\hline 1.5418 & $30-3.0(3.11-3.0)$ & $173,316 / 40,298$ & $96.9(88.7)$ & $13.3(1.5)$ & $11.5(82.1)$ \\
\hline \multicolumn{6}{|c|}{ Refinement Statistics } \\
\hline \multirow[b]{2}{*}{ Resolution (Å) } & \multirow[b]{2}{*}{ Reflections (working/test) } & \multirow[b]{2}{*}{ Total atoms } & \multirow[b]{2}{*}{$\mathbf{R}_{\text {cryst }} / \mathbf{R}_{\text {free }}{ }^{c}$} & \multicolumn{2}{|c|}{ Rmsd from ideal values } \\
\hline & & & & Bonds (Å) & Angles $\left({ }^{\circ}\right)$ \\
\hline $30-3.0$ & $38,092 / 2,007$ & 10,382 & $0.239 / 0.296$ & 0.008 & 1.27 \\
\hline \multicolumn{6}{|c|}{$\begin{array}{l}\text { Numbers in parentheses pertain to the highest resolution shell. } \\
{ }^{a}<|/ \sigma|>\text {, mean signal-to-noise, where } I \text { is the integrated intensity of a measured reflection and } \sigma \mid \text { is the estimated error in the measurement. } \\
{ }^{b} R_{\text {sym }}=100 \times \Sigma|I-<I>| / \Sigma I \text {, where } I \text { is the integrated intensity of a measured reflection. } \\
{ }^{c} R_{\text {cryst }}=\Sigma\left|F_{p}-F_{p(c a c)}\right| / \Sigma F_{p} \text {, where } F_{p} \text { and } F_{p(c a l c)} \text { are the observed and calculated structure factor amplitudes. } R_{\text {free }} \text { is calculated similarly using } \\
\text { test set reflections never used during refinement. }\end{array}$} \\
\hline
\end{tabular}


of P115-RhoGEF to enhance the activation of Rho (Wells et al., 2002). It is compelling to speculate that $\mathrm{G} \alpha 13$ stabilizes specific conformations of the $\mathrm{DH} / \mathrm{PH}$ region of P115-RhoGEF to enhance binding of Rho and subsequent nucleotide exchange.

\section{Experimental Procedures}

\section{Preparation and Crystallization}

Murine Dbs (residues 623-967), which includes both the DH and PH domains, was expressed and purified as described (Rossman et al., 2002). Protein was concentrated to $\sim 25 \mathrm{mg} / \mathrm{ml}$ in crystallization buffer $(10 \mathrm{mM}$ Tris, pH 8.0, $20 \mathrm{mM} \mathrm{NaCl}, 2 \mathrm{mM}$ DTT, $0.25 \mathrm{mM}$ EDTA) using a Vivaspin $20 \mathrm{ml}$ concentrator (Vivasciences; Hanover, Germany) with a molecular weight cut-off of $30 \mathrm{kDa}$. Dbs DH/PH was crystallized in sitting drops using vapor diffusion by combining $4 \mu \mathrm{l}$ of protein with $2 \mu \mathrm{l}$ of well solution (100 mM HEPES, $\mathrm{pH} 7.5$, $4 \%$ [v/v] ethylene glycol, $0.5 \%$ [w/v] PEG 20K). Crystals appeared within 2 days and continued to grow for several weeks. For data collection at $100 \mathrm{~K}$, crystals were transferred sequentially to well solution plus $25 \%(\mathrm{v} / \mathrm{v})$ ethylene glycol, then well solution plus $50 \%$ (v/v) ethylene glycol. The cryo-protected crystal was then suspended in a rayon loop (Hampton Research; Aliso Viejo, CA) and plunged into liquid nitrogen.

\section{Data Collection and Structure Determination}

Diffraction data were collected at the UNC X-ray facility from a single crystal using an R-AXIS IV + + area detector and a Rigaku RU-H3R rotating anode $\mathrm{X}$-ray source equipped with focusing optics. Data were integrated and scaled using DENZO and Scalepack (Otwinowski, 1993) (see Table 2). Crystals belong to space group $P 2_{1}$ with unit cell parameters $a=97.98 \AA, b=82.82 \AA, c=127.26 \AA, \beta=$ $90.8^{\circ}$. Because the monoclinic $\beta$ angle was $\approx 90^{\circ}$, we attempted to scale the data as primitive orthorhombic. However, processing and scaling the data in the primitive orthorhombic space groups resulted in rejection of $>50 \%$ of the data. Molecular replacement was accomplished using BEAST from the CCP4 suite (CCP4, 1994). A portion of the $\mathrm{DH}$ domain from the $\mathrm{Dbs} \cdot \mathrm{Cdc} 42$ structure (residues $630-814$ ) was used as the initial search model. The rotation function indicated three clear solutions, and these were sequentially positioned using the translation function. Subsequently, two $\mathrm{PH}$ domains (residues 819-945) were positioned using BEAST. Rigid body refinement using CNS (Brünger et al., 1998) was employed at this point and initial $2|\mathrm{Fo}|-|\mathrm{Fc}|$ and $|\mathrm{Fo}|-|\mathrm{Fc}|$ electron density maps were used to position the third $\mathrm{PH}$ domain using the program $\mathrm{O}$ (Jones et al., 1991). This model was then subjected to another round of rigid body refinement followed by torsion-angle simulated-annealing and positional and group temperature factor refinement. The resulting electron density maps showed clear helical density for a forth $\mathrm{DH}$ domain. Electron density maps calculated using the model including the fourth $\mathrm{DH}$ domain allowed for the positioning of the fourth $\mathrm{PH}$ domain. The final model includes 10,368 protein atoms and 14 solvent atoms with $R_{\text {cryst }}=23.9 \%\left(R_{\text {free }}=29.6 \%\right)$ using all $|F| s>0$. The mean temperature factor for all atoms is $89.4 \AA^{2}$. The Wilson temperature factor for data in the range $3.17 \AA>d>3.02 \AA$ is $92.1 \AA^{2}$. The model has good geometry with $78.7 \%$ of the residues in the core region of the Ramachandran plot with an additional $20.8 \%$ in allowed regions. Six residues $(0.5 \%)$, most of which are located in poorly ordered loops of the PH domains, occupy disallowed regions of the Ramachandran plot (see Table 2). Because of crystal disorder, the four molecules have been modeled to different extents. Molecule A includes residues $624-844,854-877$, and 887958; molecule B, residues 624-845, 852-878, and 885-966; molecule $\mathrm{C}$, residues $624-842,857-878$, and $883-950$; and molecule D, residues 624-877 and 889-952. In addition, several residues in poorly defined regions of the structure have been modeled as alanines.

\section{Acknowledgments}

We thank Nicolas Nassar for sharing the coordinates of Trio DH/ $\mathrm{PH}$ prior to publication. This work was supported by the National
Institutes of Health grant R01-GM62299 (J.S.) and the American Cancer Society Fellowship PF-00-163-01-GMC (D.K.W.)

Received: January 28, 2004

Revised: March 24, 2004

Accepted: March 25, 2004

Published: June 8, 2004

\section{References}

Aghazadeh, B., Zhu, K., Kubiseski, T.J., Liu, G.A., Pawson, T., Zheng, Y., and Rosen, M.K. (1998). Structure and mutagenesis of the Dbl homology domain. Nat. Struct. Biol. 5, 1098-1107.

Aghazadeh, B., Lowry, W.E., Huang, X.Y., and Rosen, M.K. (2000). Structural basis for relief of autoinhibition of the Dbl homology domain of proto-oncogene Vav by tyrosine phosphorylation. Cell 102, 625-633.

Baumeister, M.A., Martinu, L., Rossman, K.L., Sondek, J., Lemmon, M.A., and Chou, M.M. (2003). Loss of phosphatidylinositol 3-phosphate binding by the $\mathrm{C}$-terminal Tiam-1 pleckstrin homology domain prevents in vivo Rac1 activation without affecting membrane targeting. J. Biol. Chem. 278, 11457-11464.

Brünger, A.T., Adams, P.D., Clore, M.G., DeLano, W.L., Gros, P., Grosse-Kunstleve, R.W., Jiang, J.-S., Kuszewski, J., Nilges, M., Pannu, N.S., et al. (1998). Crystallography \& NMR system: a new software suite for macromolecular structure determination. Acta Crystallogr. D 54, 905-921.

CCP4 (Collaborative Computational Project 4) (1994). The CCP4 suite: programs for protein crystallography. Acta Crystallogr. D 50 , 760-763.

Christopher, J.A. (1998). SPOCK: the Structural Properties Observation and Calculation Kit. The Center for Macromolecular Design, Texas A\&M University, College Station, TX.

Crompton, A.M., Foley, L.H., Wood, A., Roscoe, W., Stokoe, D., McCormick, F., Symons, M., and Bollag, G. (2000). Regulation of Tiam1 nucleotide exchange activity by pleckstrin domain binding ligands. J. Biol. Chem. 275, 25751-25719.

Etienne-Manneville, S., and Hall, A. (2002). Rho GTPases in cell biology. Nature 420, 629-635.

Eva, A., Vecchio, G., Rao, C.D., Tronick, S.R., and Aaronson, S.A (1988). The predicted DBL oncogene product defines a distinct class of transforming proteins. Proc. Natl. Acad. Sci. USA 85, 2061-2065. Ferguson, K.M., Kavran, J.M., Sankaran, V.G., Fournier, E., Isakoff, S.J., Skolnik, E.Y., and Lemmon, M.A. (2000). Structural basis for discrimination of 3-phosphoinositides by pleckstrin homology domains. Mol. Cell 6, 373-384.

Han, J., Luby-Phelps, K., Das, B., Shu, X., Xia, Y., Mosteller, R.D. Krishna, U.M., Falck, J.R., White, M.A., and Broek, D. (1998). Role of substrates and products of PI 3-kinase in regulating activation of Rac-related guanosine triphosphatases by Vav. Science 279, 558-560.

Innocenti, M., Tenca, P., Frittoli, E., Faretta, M., Tocchetti, A., Di Fiore, P.P., and Scita, G. (2002). Mechanisms through which Sos-1 coordinates the activation of Ras and Rac. J. Cell Biol. 156, 125-136.

Jones, T.A., Zou, J.Y., Cowan, S.W., and Kjeldgaard, M. (1991). Improved methods for building protein models in electron density maps and location of errors in these models. Acta Crystallogr. A 47, 110-119.

Kraulis, P. (1991). MOLSCRIPT: a program to produce both detailed and schematic plots of protein structures. J. Appl. Crystallogr. 24, 946-950.

Kubiseski, T.J., Culotti, J., and Pawson, T. (2003). Functional analysis of the Caenorhabditis elegans UNC-73B PH domain demonstrates a role in activation of the Rac GTPase in vitro and axon guidance in vivo. Mol. Cell. Biol. 23, 6823-6835.

Lemmon, M.A., and Ferguson, K.M. (2000). Signal-dependent membrane targeting by pleckstrin homology $(\mathrm{PH})$ domains. Biochem. J. 350, 1-18.

Liu, X., Wang, H., Eberstadt, M., Schnuchel, A., Olejniczak, E.T., 
Meadows, R.P., Schkeryantz, J.M., Janowick, D.A., Harlan, J.E., Harris, E.A., et al. (1998). NMR structure and mutagenesis of the $\mathrm{N}$-terminal Dbl homology domain of the nucleotide exchange factor Trio. Cell 95, 269-277.

Merritt, E.A., and Murphy, M.E.P. (1994). Raster3D Version 2.0. A program for photorealistic molecular graphics. Acta Crystallogr. D $50,869-873$.

Nimnual, A., and Bar-Sagi, D. (2002). The two hats of SOS. Sci STKE 2002, PE36.

Nimnual, A.S., Yatsula, B.A., and Bar-Sagi, D. (1998). Coupling of Ras and Rac guanosine triphosphatases through the Ras exchanger Sos. Science $279,560-563$.

Otwinowski, Z. (1993). Data Collection and Processing (Warrington, UK: Daresbury Laboratory).

Ridley, A.J. (2001). Rho family proteins: coordinating cell responses. Trends Cell Biol. 11, 471-477.

Rossman, K.L., Worthylake, D.K., Snyder, J.T., Siderovski, D.P., Campbell, S.L., and Sondek, J. (2002). A crystallographic view of interactions between Dbs and Cdc42: $\mathrm{PH}$ domain-assisted guanine nucleotide exchange. EMBO J. 21, 1315-1326.

Rossman, K.L., Cheng, L., Mahon, G.M., Rojas, R.J., Snyder, J.T., Whitehead, I.P., and Sondek, J. (2003). Multifunctional roles for the $\mathrm{PH}$ domain of Dbs in regulating Rho GTPase activation. J. Biol. Chem. 278, 18393-18400.

Russo, C., Gao, Y., Mancini, P., Vanni, C., Porotto, M., Falasca, M., Torrisi, M.R., Zheng, Y., and Eva, A. (2001). Modulation of oncogenic DBL activity by phosphoinositol phosphate binding to pleckstrin homology domain. J. Biol. Chem. 276, 19524-19531.

Scita, G., Nordstrom, J., Carbone, R., Tenca, P., Giardina, G., Gutkind, S., Bjarnegard, M., Betsholtz, C., and Di Fiore, P.P. (1999). EPS8 and E3B1 transduce signals from Ras to Rac. Nature 401, 290-293.

Scita, G., Tenca, P., Areces, L.B., Tocchetti, A., Frittoli, E., Giardina, G., Ponzanelli, I., Sini, P., Innocenti, M., and Di Fiore, P.P. (2001). An effector region in Eps8 is responsible for the activation of the Rac-specific GEF activity of Sos-1 and for the proper localization of the Rac-based actin-polymerizing machine. J. Cell Biol. 154, 1031-1044.

Snyder, J.T., Worthylake, D.K., Rossman, K.L., Betts, L., Pruitt, W.M., Siderovski, D.P., Der, C.J., and Sondek, J. (2002). Structural basis for the selective activation of Rho GTPases by Dbl exchange factors. Nat. Struct. Biol. 9, 468-475.

Soisson, S.M., Nimnual, A.S., Uy, M., Bar-Sagi, D., and Kuriyan, J. (1998). Crystal structure of the Dbl and pleckstrin homology domains from the human Son of sevenless protein. Cell 95, 259-268.

Wells, C.D., Liu, M.Y., Jackson, M., Gutowski, S., Sternweis, P.M., Rothstein, J.D., Kozasa, T., and Sternweis, P.C. (2002). Mechanisms for reversible regulation between $\mathrm{G} 13$ and Rho exchange factors. J. Biol. Chem. 277, 1174-1181.

Whitehead, I., Kirk, H., Tognon, C., Trigo-Gonzalez, G., and Kay, R. (1995). Expression cloning of Ifc, a novel oncogene with structural similarities to guanine nucleotide exchange factors and to the regulatory region of protein kinase C. J. Biol. Chem. 270, 18388-18395. Whitehead, I.P., Campbell, S., Rossman, K.L., and Der, C.J. (1997). Dbl family proteins. Biochim. Biophys. Acta 1332, F1-23.

Whitehead, I.P., Lambert, Q.T., Glaven, J.A., Abe, K., Rossman, K.L., Mahon, G.M., Trzaskos, J.M., Kay, R., Campbell, S.L., and Der, C.J. (1999). Dependence of Dbl and Dbs transformation on MEK and NFkappaB activation. Mol. Cell. Biol. 19, 7759-7770.

Worthylake, D., Rossman, K., and Sondek, J. (2000). Crystal structure of Rac1 in complex with the guanine nucleotide exchange region of Tiam1. Nature 408, 682-688.

\section{Accession Numbers}

The coordinates have been deposited in the protein data bank under ID code 1RJ2. 